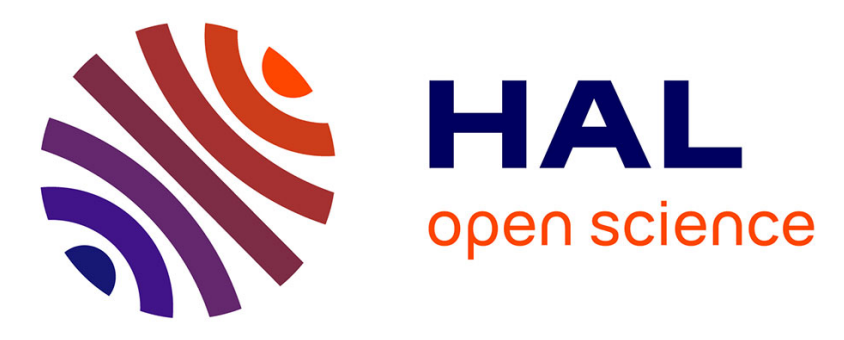

\title{
Rho-specific Guanine nucleotide exchange factors (Rho-GEFs) inhibition affects macrophage phenotype and disrupts Golgi complex
}

Wei Chen, Yue Zhao, Xian C. Li, Jacek Z. Kubiak, Rafik M. Ghobrial, Malgorzata Kloc

\section{To cite this version:}

Wei Chen, Yue Zhao, Xian C. Li, Jacek Z. Kubiak, Rafik M. Ghobrial, et al.. Rho-specific Guanine nucleotide exchange factors (Rho-GEFs) inhibition affects macrophage phenotype and disrupts Golgi complex. International Journal of Biochemistry and Cell Biology, 2017, 93, pp.12-24. 10.1016/j.biocel.2017.10.009 . hal-01647190

HAL Id: hal-01647190

https://hal-univ-rennes1.archives-ouvertes.fr/hal-01647190

Submitted on 4 Dec 2017

HAL is a multi-disciplinary open access archive for the deposit and dissemination of scientific research documents, whether they are published or not. The documents may come from teaching and research institutions in France or abroad, or from public or private research centers.
L'archive ouverte pluridisciplinaire HAL, est destinée au dépôt et à la diffusion de documents scientifiques de niveau recherche, publiés ou non, émanant des établissements d'enseignement et de recherche français ou étrangers, des laboratoires publics ou privés. 
Revised Manuscript \# BC-D-17-00414 R1

Rho-specific Guanine nucleotide exchange factors (Rho-GEFs) inhibition affects macrophage phenotype and disrupts Golgi complex

Wei Chen ${ }^{1,2}$, Yue Zhao ${ }^{1}$, Xian C Li ${ }^{1,3}$, Jacek Z. Kubiak ${ }^{4,5,6}$, Rafik M Ghobrial1,3, Malgorzata $\mathrm{Kloc}^{\star 1,3,7}$

${ }^{1}$ The Houston Methodist Research Institute, Houston, Texas, USA;

${ }^{2}$ Department of Nephrology, Second Xiangya Hospital, Central South University, Changsha 410011, China.

${ }^{3}$ The Houston Methodist Hospital, Department of Surgery, Houston, Texas, USA;

${ }^{4}$ CNRS UMR 6290, Institute of Genetics and Development of Rennes, Cell Cycle Group, IFR 140 GFAS, France;

${ }^{5}$ University of Rennes 1, Faculty of Medicine, Rennes, France;

${ }^{6}$ Department of Regenerative Medicine, Military Institute of Hygiene and Epidemiology (WIHE), Warsaw, Poland;

${ }^{7}$ The University of Texas, MD Anderson Cancer Center, Department of Genetics, Houston Texas, USA

*Correspondence Address:

Malgorzata Kloc

The Houston Methodist Hospital, Department of Surgery,

6550 Fannin St., Houston, TX 77030

Tel. 713.441 .6875

Fax 713.790 .3755

e-mail: mkloc@houstonmethodist.org

Graphical abstract 


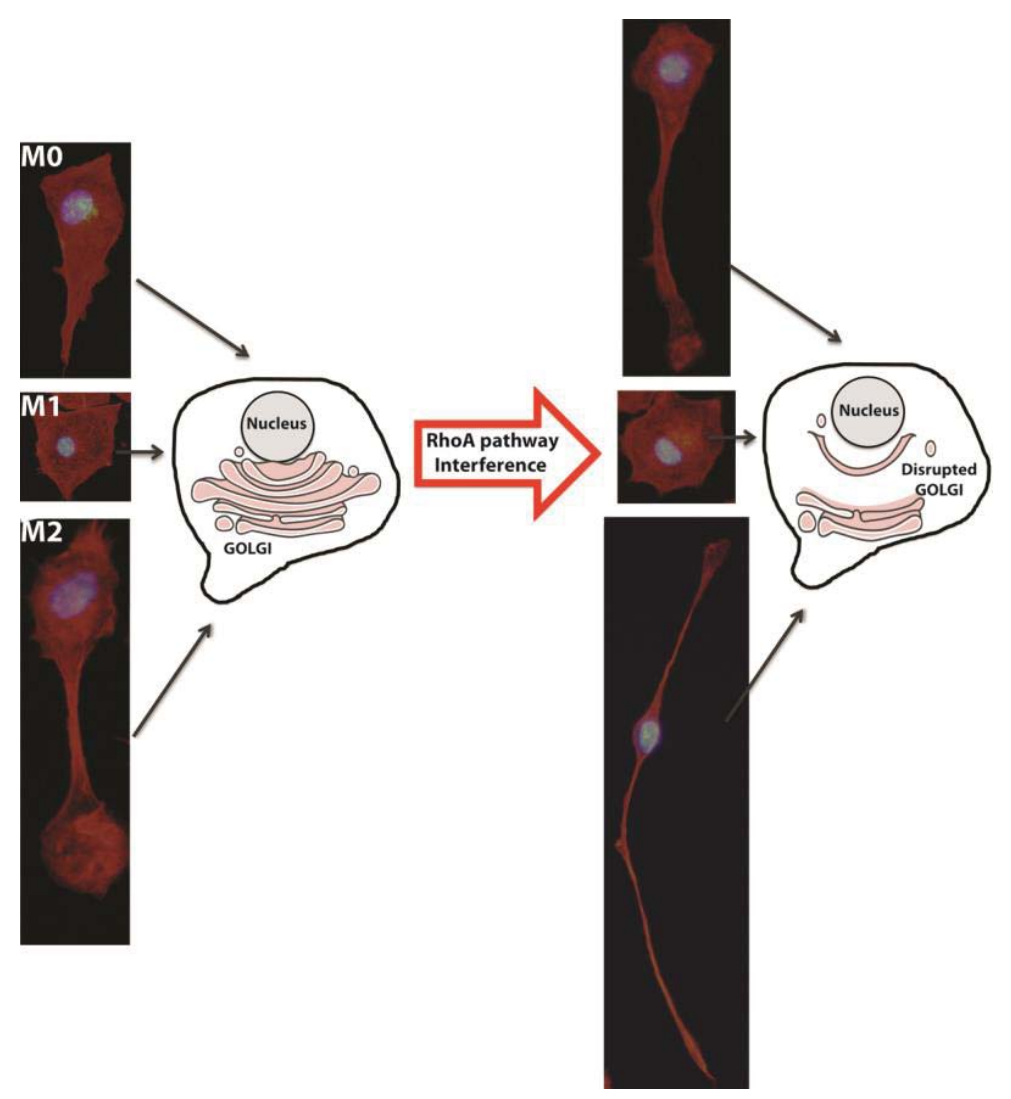

\section{Highlights}

1.GEFs inhibition affects $M O$ and M2 macrophage phenotype

2.GEFs inhibition does not change the phenotype of M1 macrophages

3.GEFs inhibition and RhoA deletion disrupts ultrastructure of macrophage Golgi complex

\section{Abstract}

Macrophages play crucial role in tissue homeostasis and the innate and adaptive immune response. Depending on the state of activation macrophages acquire distinct phenotypes that depend on actin, which is regulated by small GTPase RhoA. The naive MO macrophages are slightly elongated, pro-inflammatory M1 are round and M2 antiinflammatory macrophages are elongated. We showed previously that interference with 
RhoA pathway (RhoA deletion or RhoA/ROCK kinase inhibition) disrupted actin, produced extremely elongated (hummingbird) macrophage phenotype and inhibited macrophage movement toward transplanted hearts. The RhoA function depends on the family of guanine-nucleotide exchange factors (GEFs), which catalyze the exchange of GDP for GTP and activate RhoA that reorganizes actin cytoskeleton. Using actin staining, immunostaining, Western blotting, flow cytometry and transmission electron microscopy we studied how a direct inhibition of Rho-GEFs with Rhosin (Rho GEFbinding domain blocker) and Y16 (Rho GEF DH-PH domain blocker) affects MO, M1 and M2 macrophage phenotypes. We also studied how Rho-GEFs inhibition and RhoA deletion affects organization of Golgi complex that is crucial for normal macrophage functions such as phagocytosis, antigen presentation and receptor recycling. We found that GEFs inhibition differently affected MO, M1 and M2 macrophages phenotype and that GEFs inhibition and RhoA deletion both caused changes in the ultrastructure of the Golgi complex. These results suggest that actin/ RhoA- dependent shaping of macrophage phenotype has different requirements for activity of RhoA/GEFs pathway in MO, M1 and M2 macrophages, and that RhoA and Rho-GEFs functions are necessary for the maintenance of actin-dependent organization of Golgi complex.

\section{Abbreviations}

Arg1- Arginase 1

DH-PH domain- The diffuse B-cell lymphoma (Dbl) domain and pleckstrin-homology domain

GDP-Guanosine diphosphate

GEF- Guanine exchange factor

GM130-130 kDa cis Golgi matrix protein

GTP- Guanosine triphosphate 
IFN- $\mathrm{Y}$ - Interferon $\mathrm{Y}$

iNos-Inducible nitric oxide synthase

IL-4- Interleukin 4

IL-13- Interleukin 13

LARG- leukemia-associated Rho guanine nucleotide exchange factor

LPS- Lipopolysaccharides

p115- peripheral membrane protein

PDZ domain- PDZ name is a combination of the first letters of the first three proteins

discovered to share this domain - post synaptic density protein (PSD95), Drosophila disc large tumor suppressor (Dlg1), and zonula occludens-1 protein (zo-1)

RhoA- Ras homolog gene family, member A

ROCK- Rho-associated, coiled-coil-containing protein kinase 1

Y16- Rho inhibitor II

Keywords: macrophage, actin, cytoskeleton, Golgi complex, RhoA

\section{Introduction}

The macrophages are extremely heterogeneous both phenotypically and functionally. The classical macrophage subtypes are naïve/unactivated MO, pro-inflammatory M1, and anti-inflammatory M2 macrophages (Geissmann et al., 2010; Hettinger et al., 2013; Murray and Wynn, 2011). Others and we showed that these three macrophage subtypes have very distinct shapes: MO are slightly elongated, M1 are roundish, pancake-like, and M2 are elongated (Geissmann et al., 2010; Hettinger et al., 2013; Liu et al 2016a,b,c; Murray and Wynn, 2011). Macrophage shape, functions and organization of their subcellular organelles such as the Golgi complex depend on actin cytoskeleton that is regulated by small GTPase RhoA pathway (Bruce et al, 2015; Egea 
et al., 2015; Kloc et al. 2014; Maxfield and McGraw, 2004; Mori et al., 1994; Prosser and Wendland, 2012; Rossanese et al, 2001; Wheeler and Ridley, 2004; Zilberman et al., 2011). We showed previously that genetic (RhoA deletion) or pharmacologic (RhoA/ROCK kinase inhibition) interference with RhoA pathway disrupts actin cytoskeleton, causes extreme elongation (hummingbird phenotype) of MO and M2 macrophages, prevents macrophage accumulation within the graft and inhibits chronic rejection of transplanted rodent hearts (Kloc et al., 2014; Liu et al 2016 a,b,c; Liu et al., 2017). It has been shown that activation of RhoA depends on the guanine nucleotide exchange factors (GEFs) that catalyze the exchange of GDP for GTP (Bos et al., 2007; Cherfils and Zeghouf, 2013; Gilman, 1987). The activity of many GEFs depends on the tandem Dbl homology (DH), pleckstrin-homology $(\mathrm{PH})$ and/or PDZ catalytic domains that facilitate GDP for GTP exchange (Shang et al., 2013). Here we used two different GEFs inhibitors: the Y16 that blocks RhoGEF DH-PH domain junction and prevents Rho GEFs LARG, p115, and PDZ interaction with RhoA, and rhosin that directly targets Rho GEF binding domain preventing Rho from interacting with its GEFs (Dubash et al. 2007; Shang et al., 2013, 2014) to study how Rho-GEFs inhibition affects MO, M1 and M2 macrophage phenotype. Our previous studies showed that RhoA pathway interference inhibited phagocytosis and affected macrophage receptor recycling (Liu et al., 2016b; Liu et al., 2017). Because both these processes depend on proper functioning and structure of the Golgi (known to be matrix protein- and actin-dependent; Bruce et al., 2015; Prosser and Wendland, 2012; Seemann et al., 2000), here we also studied the effect of RhoA deletion and Rho-GEFs inhibition on the organization of macrophage Golgi complex.

\section{Results}

\section{Effect of Rho-GEFs inhibition on shape of peritoneal macrophages}


We polarized peritoneal MO macrophages using LPS and IFN-Y into M1, or using IL-4 and IL-13 into M2 phenotype, in absence or presence of either rhosin or Y16 GEFs inhibitor. We found that while untreated unactivated $\mathrm{MO}$ macrophages were slightly elongated with well-defined front (containing nucleus) and tail (Fig.1A), the macrophages polarized into M1 in the absence of GEFs inhibitors were round (Fig.1B), and those polarized into M2 were elongated (Fig.1C). Incubation of MO macrophages with rhosin alone or $\mathrm{Y} 16$ alone resulted in dramatic change of $\mathrm{MO}$ phenotype; they became either elongated M2-like or extremely elongated and multipolar (Fig.1A1, A2; Fig 2, Fig.3). In contrast, polarization with LPS and IFN-y in the presence of rhosin or Y16 had no or much less effect on phenotype of M1 macrophages; they remained roundish or slightly elongated/ multipolar (Fig.1B1, B2; Fig. 2). Polarization with IL-4 and IL-13 in the presence of rhosin or Y16 caused extreme elongation and multipolarity of M2 macrophages, or rounding into M1-like phenotype (Fig. C1a, C1b, C2a, C2b; Fig. 2, Fig. 3). The quantification of the effect of inhibitors on macrophage shape is presented in Fig. 2 and Fig. 3 and summary of macrophage shape changes is presented in Fig. 4. Each experiment was repeated 3-5 times. For quantification analysis of phenotype frequency we combined numbers of cells with given phenotype calculated in three separate slides from three independent experiments within five different $0.05 \mathrm{~mm}^{2}$ area on each slide. The consistency between the results from three different repeats of the experiments is exemplified by the graphs with $P$ values in Fig.3D, E. The number of cells was calculated under $20 \mathrm{X}$ objective in two different $15 \mathrm{~mm}^{2}$ areas of the slide in each experiment. This graphs show that there is no statistically significant difference between the experiments.

Effect of Rho-GEFs inhibition on expression of M1 and M2 macrophage molecular markers 
We wanted to see if the phenotypic changes imposed on macrophages by GEFs inhibition affected the expression of subtype-specific macrophage markers. We studied the expression of iNos and Arg1 proteins, which are molecular markers of M1 and M2 macrophages, respectively. Both Western blot (Fig. 5) and flow cytometry (data not shown) analyses indicated that there is no statistically significant difference in iNos and Arg1 expression level between untreated and rhosin or $\mathrm{Y} 16$ treated macrophages.

\section{Rho-GEFs inhibition, similar to RhoA deletion, disrupts Golgi complex}

We used immunostaining with antibody against Golgi matrix protein GM130 $(23,24)$ that plays a role in the stacking of Golgi cisternae and in the vesicular transport, and the transmission electron microscopy to assess Golgi distribution, morphology and ultrastructure in control and Rho-GEFs inhibitors treated wild type and RhoA-deleted $\mathrm{MO}, \mathrm{M} 1$ and M2 macrophages.

\section{Immunostaining}

Immunostaing with GM130 antibody showed that in untreated wild type MO, M1 and M2 macrophages Golgi complex formed a compact body built of tightly apposed cisternae in the vicinity of the nucleus (Fig. 6A-C). Treatment with Rho-GEFs inhibitors caused four distinct, observable at light microscopy level, effects: decrease in the size of compact Golgi, loosening (unwinding) of Golgi ribbons (collections of Golgi stacks), relocation away from the nucleus and spreading of the GM130 positive signal in larger area of macrophage. In MO and M1 macrophages treatment with rhosin or $\mathrm{Y} 16$ caused diminution of compact Golgi and dispersion of GM130 staining around remnants of compact Golgi (Fig. 6A1, A2 and B1, B2). In M2 macrophages rhosin and Y16 treatment also resulted in less compact Golgi and more dispersed GM130 staining, and in extremely elongated M2 macrophages translocation of GM130 positive staining to the macrophage tail (Fig.6C1-C3). We quantified the effect of inhibitors on distribution of 
GM130 staining (Golgi compactness/dispersion) by calculating the diameter/lenght of the area of staining in the vicinity of the nucleus (Fig.8). While in control macrophages the area occupied by GM130 staining was very compact and spherical and its diameter was equal to $5.73 \pm 0.89 \mu \mathrm{m}$ in rhosin treated and $\mathrm{Y} 16$ treated macrophages the area was much les compact and its length equaled to10.55 $\pm 3.77 \mu \mathrm{m}$ and $13.49 \pm 6.24 \mu \mathrm{m}$, respectively. The difference in the compactness/dispersion of GM130 staining between control and rhosin- or Y16-treated macrophages was statistically significant with $p<0.01$, and the difference between rhosin- and Y16-treatement was statistically insignificant (Fig.8A).

Although the RhoA deletion in macrophages also affected the structure of the Golgi complex the effect of RhoA deletion was slightly different than the effect of Rho-GEFs inhibition. The GM130 staining of Rho KO macrophages showed two major effects observable at light microscopy level: diminution of the size of compact Golgi and translocation of the GM130 staining far away from the nucleus. In RhoA-deleted MO and M1 macrophages compact Golgi was smaller than in the wild type macrophages (Fig.7A, B; Fig.8B). In RhoA KO M2 macrophages the dispersed GM130 staining was visible in the cell body and also distributed in the macrophage tail, front and within the filopodia (Fig.7C, D). Fig. 8 shows that the area of compact GM130 staining in RhoA deleted macrophages was statistically significantly smaller than in control macrophages (control: $5.73 \pm 0.89 \mu \mathrm{m}$ versus RhoA KO: $3.98 \pm 1.26 \mu \mathrm{m}$, with $\mathrm{p}<0.01$ ). For quantification analysis Golgi staining, we combined length of the Golgi staining calculated in three separate slides from three independent experiments within five different $0.05 \mathrm{~mm}^{2}$ area on each slide.

\section{Electron microscopy: effect of Rho-GEFs inhibition and RhoA deletion on Golgi utrastructure}


Because the immunostaining only confirms the presence and location of GM130 protein but doesn't show if there are any changes in Golgi ultrastructure, we performed electron microscopy study. Ultrastructural analysis of approximately 100 cells in each sample showed that in untreated wild type M0, M1 and M2 macrophages the Golgi complex consisted of multiple stacks of orderly arranged membranous cisternae located in the vicinity of the nucleus (Fig.9A). In contrast, the RhoA deletion, and Y16 or rhosin treatment caused dramatic reduction or complete obliteration of the Golgi stacks and translocation of the Golgi remnants away from the cell nucleus (Fig.9B-D). We measured the distance (in $\mathrm{nm}$ ) between the nucleus and Golgi cisternae and found that while in wild type macrophages Golgi was situated at $309.18 \pm 184.2 \mathrm{~nm}$ distance from the nucleus, in RhoA deleted macrophages this distance was over 4 times larger i.e. $1305.95 \pm 558.6 \mathrm{~nm}$. This difference was statistically significant with $p<0.0001$ (Fig.8C) For electron microscopy analysis each grid from each experiment contained 100 cells. The cells were examined at electron microscopy level and 2-3 representative cells out of 100 from each grid were used for the measurements. The distance between nuclear membrane and first recognizable Golgi cisternae was measured on electron microscopy images. Although by immunostaining we did see the GM130 positive staining in M2 macrophage tail, we have not identified ultrastructurally recognizable Golgi cisternae in this location. This may indicate that either the GM130 protein remained associated with ultrastructurally unrecognizable fragments of Golgi or that the GM130 protein dissociated from the remnants of the dissipating Golgi. It is known that Golgi complex is associated with the pair of centrioles, which are a component of MTOC that, among variety of functions, plays important role in immunological response (Kloc et al. 2014). Electron microscopy analysis showed that while in the wild type macrophages centrioles are surrounded by or are in close vicinity of the Golgi cisternae (Fig.10A,B) in RhoAdeleted macrophages the centrioles are surrounded by the cytoplasm containing 
remnants of Golgi (Fig.10C,D). In summary, all these data indicate that RhoA/GEFs activity is needed for the maintenance of intact structure of the Golgi complex.

\section{Discussion}

RhoA is a small GTPase, which, through its downstream effectors such as ROCK p160 kinase, regulates actin filament polymerization and organization, and through actin regulates cell movement, phagocytosis, matrix degradation and receptor recycling in eukaryotic cells (Wheeler and Ridley, 2004). Similar to other GTPases, the RhoA is active in GTP-bound and inactive in GDP-bound state. The process of GDP to GTP exchange is facilitated by the guanine exchange factors GEFs. The binding of GEFs to GTPase promotes dissociation of GDP replacing it with GTP. There are multiple GEFs with different functions. Some GEFs for Rho GTPases contain two adjacent domains, which together are responsible GEF activity: the Dbl homology DH domain (RhoGEF domain) and the pleckstrin homology PH domain (Bos et al. 2007; Cherfils and Zeghouf, 2013). Other, such as leukemia-associated Rho GEF (LARG), p115, and PDZ may also contain a proline-rich PSD-95/DlgA/ZO-1 PDZ domain that is responsible for proteinprotein interactions (Bos et al. 2007; Cherfils and Zeghouf, 2013).

We showed that Rho-GEFs inhibition using Y16, which blocks Rho GEF DH-PH domain junction and prevents Rho GEFs LARG, p115, and PDZ interaction with RhoA, and rhosin, which directly targets Rho GEF binding domain preventing Rho from interacting with its GEFs (Fig. 10), has, similar to RhoA deletion, profound effect on macrophage shape causing extreme elongation of MO and M2 macrophages (Fig. 10 and 11; Liu et al., 2016 a, b, c). This indicates that MO and M2 macrophage shape, in contrast to M1 shape, is vulnerable to Rho-GEFs inhibition. Similar vulnerability of MO and M2 phenotype and resistance of M1 phenotype was also observed in RhoA-interfered macrophages (Kloc et al., 2014; Liu et al., 2016 a, b, c; Liu et al., 2017). Several studies showed that mechanically enforced elongation of macrophages into M2-like type 
induces expression of M2 specific markers in MO macrophages (Lee et al., 2016; McWhorter et al., 2013; Wang et al., 2016). In contrast, we showed here that GEFs inhibition-induced elongation of MO into M2-like phenotype was insufficient to induce expression of M2 specific marker Arg1. This indicates that mechanically enforced elongation operates differently than elongation caused by genetic (RhoA deletion) or inhibitor-induced elongation. Further studies are needed to define commonalities and differences between these two modes of macrophage elongation. It has been shown recently that cell elongation depends on evolutionary conserved "elongation mechanism" that combines equatorial constriction of the cell by an actomyosin ring, with actin-driven contractions of the cytoplasm that help transform cellular belt-tightening into cellular stretch (Hoff, 2014; Lee et al., 2016). It seems that when macrophage is already initially elongated (MO and M2 phenotype), and the RhoA-dependent "elongation mechanism" is already operational, it is easy to induce further elongation (MO elongation into M2-like or extreme elongation of M2). In contrast, it seems that when initially elongated MO macrophage acquires M1 "round" phenotype the "elongation mechanism" becomes completely and nearly irreversibly shut off. Another, very likely possibility is that the "round phenotype" of M1 macrophages is mainly RhoAindependent. Although phenomenon of cell rounding in mitosis is known to be dependent on actin cell cortex and regulated by Cyclin-dependent kinase 1, Cdk1; Clark and Paluch, 2011), and cell rounding of human endothelial cells depends on actin filaments and $\mathrm{Ca}^{+}$influx (Lin et al., 2016) further studies are needed to clarify what molecules/pathways regulate M1-specific phenotype in macrophages.

We also showed that RhoA deletion and Rho-GEFs inhibition caused disruption of normal ultrastructure of Golgi complex in MO, M1 and M2 macrophages. Macrophage functions such as phagocytosis, antigen presentation and receptor recycling to and from the cell membrane all depend on properly structured Golgi complex (Bruce et al., 2015; 
Marra et al., 2007; Nakamura, 2010; Prosser and Wendland, 2012). Thus, our present data showing the disruption of Golgi structure upon RhoA deletion or Rho-GEFs inhibition explain our previous findings that RhoA pathway interference inhibits macrophage phagocytosis and receptor recycling (Fig.11; Kloc et al., 2014, Liu et al., 2017).

\section{Material and Methods}

Breeding and all experiments were performed according to The Methodist Hospital Research Institute's animal care and use NIH standards in concordance with the "Guide for the Care and Use of Laboratory Animals" (DHHS publication No. (NIH) 85-23 Revised 1985), the PHS "Policy on Humane Care and Use of Laboratory Animals" and the NIH "Principles for the Utilization and Care of Vertebrate Animals Used in Testing, Research and Training." All studies were performed according to Houston Methodist Institutional Animal Care and Use Committee animal protocol AUP-0317-0006 (IS00003962) entitled "Tolerance Induction in a Rodent System". Mice euthanasia was performed according to TMHRI Euthanasia of Rodents Procedure, by isoflurane overdose via a vaporizer inhalation followed by cervical dislocation and thoracotomy to ensure the death. For the genotyping mice were anesthetized with 3-4 \% isoflurane. RhoA ${ }^{\text {flox/flox }}$ mice (gift from dr Richard A. Lang from UC Department of Pediatrics and UC Department of Ophthalmology, Cincinnati Children's Hospital, Cincinnati, Ohio) were crossbred with B6.129P2-Lyz2tm1(cre)lfo/J mice (JAX® Mice (Bar Harbor, Maine, USA)

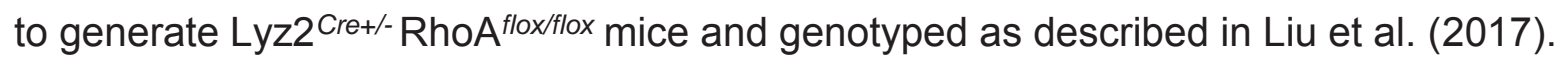

\section{Peritoneal macrophages}

Mouse peritoneal macrophages from no Cre mice (Lyz2 ${ }^{\text {Cre-l- }}$ RhoA $A^{\text {flox/flox }}$ ), further called the wild type or RhoA deleted Lyz2 ${ }^{\mathrm{Cre}+/-}$ RhoA ${ }^{\text {flox/flox }}$ mice, forther called the KO were purified by overnight incubation in Dulbecco's Modified Eagle Medium (DMEM) with $10 \%$ fetal bovine serum (FBS), $100 \mathrm{U} / \mathrm{ml}$ penicillin and100 mg/ml streptomycin (all from 
Thermo Fisher Scientific, Waltham, MA, USA) and subsequent removal of free-floating cells. For M1 polarization, macrophages were incubated for $24 \mathrm{hr}$ either with $20 \mathrm{ng} / \mathrm{ml}$ of recombinant murine IFN-y (Peprotech, Rocky Hill, NJ, USA) and100ng/ml of Lipopolysaccharide (LPS) (Sigma Aldrich,St. Louis, MO, USA) or for M2 polarization, with $20 \mathrm{ng} / \mathrm{ml}$ of recombinant murine IL-4 (Peprotech) and $10 \mathrm{ng} / \mathrm{ml}$ of recombinant murine IL-13 (Peprotech) as described previously (Liu et al, 2016a.b). Macrophages were grown either in 6/12 well plastic petri dishes or in glass chamber slides. Immunostaining, flow cytometry and Transmission Electron Microscopy Macrophages were seeded on chamber slides (for immunostaining) and/or in 12 well plates (for TEM). For immunostaining macrophages were fixed in $4 \%$ formaldehyde in PBS with $0.05 \%$ Triton X100. After washing in PBS-Tween 20 fixed cells were blocked in Casein Blocking buffer (BioRad, USA) with 0.05\% Tween 20 and subsequently incubated overnight, at $4^{\circ} \mathrm{C}$, in blocking buffer with 1:200 dilution of anti-GM130 (Cterminal) antibody from Sigma Aldrich. After extensive washing in PBS-Tween 20, cells were incubated in blocking buffer with 1:200 dilution of FITC -conjugated secondary antibody from ThermoFisher Scientific with or without Rhodamine-phalloidin $(2 \mu$ of methanolic solution $/ 500 \mathrm{ml}$ ) for several hours at room temperature. After extensive washing in PBS-Tween 20 cells were mounted in ProLong® Gold Antifade with DAPI from Fisher Scientific and observed under Nikon fluorescence microscope.

For electron microscopy cell were fixed in $2 \%$ formaldehyde, $3 \%$ glutaraldehyde (both EM grade from Ted Pella Inc.) in 1x PBS. Fixed cells were washed in $0.1 \mathrm{M}$ sodium cacodylate buffer and treated with $0.1 \%$ Millipore-filtered cacodylate buffered tannic acid, postfixed with $1 \%$ buffered osmium, and stained en bloc with $1 \%$ Milliporefiltered uranyl acetate. The samples were dehydrated in increasing concentrations of ethanol, infiltrated, and embedded in LX-112 medium. The samples were polymerized in a $60 \mathrm{C}$ oven for approximately 3 days. Ultrathin sections were cut using an Ultracut 
microtome (Leica, Deerfield, IL), stained with uranyl acetate and lead citrate in a Leica EM Stainer, and examined in a JEM 1010 transmission electron microscope (JEOL, USA, Inc., Peabody, MA) at an accelerating voltage of $80 \mathrm{kV}$. Digital images were obtained using AMT Imaging System (Advanced Microscopy Techniques Corp, Danvers, MA). Flow cytometry and Western blotting were performed as described previously (Liu et al., 2017). All experiments were repeated 3-6 times. For quantification analysis of phenotype frequency and Golgi staining, we combined numbers of cells or length of the Golgi staining calculated in three separate slides from three independent experiments within five different $0.05 \mathrm{~mm}^{2}$ area on each slide. The consistency between the results from three different repeats of the experiments was assured by the calculation of the number of cells with given phenotype under 20X objective in two different $15 \mathrm{~mm}^{2}$ areas of the slide in each experiment.

Conflicts of interest: none of the authors has conflict of interest

\section{Authors Contributions}

Wei Chen - performed macrophage isolation, polarization, Flow cytometry and immunostaining

Yue Zhao- performed quantifications and statistical analyses

Xian C Li, Jacek Z. Kubiak and Rafik M Ghobrial- analyzed data

Malgorzata Kloc- performed immunostaining and electron microscopy, analyzed data and wrote the manuscript

\section{Acknowledgements}

We are grateful for the support from William Stamps Farish Fund and Donald D. Hammill Foundation. We also thank Kenneth Dunner Jr. for electron microscopy work, 
and acknowledge CCSG grant NIH P30CA016672 for MDACC High Resolution Electron Microscopy Facility. 


\section{References}

1. Bos JL, Rehmann H, Wittinghofer A. (2007). GEFs and GAPs: critical elements in the control of small G proteins. Cell. 129: 865-877.

2. Bruce L. Goode BL, Eskin JA, Wendland B (2015). Actin and Endocytosis in Budding Yeast Genetics. 199: 315-358.

3. Cherfils J; Zeghouf M. (2013). Regulation of small GTPases by GEFs, GAPs, and GDIs. Physiological reviews, 93: 269-309.

4. Clark AG and Paluch E. (2011) Mechanics and Regulation of Cell Shape During the Cell Cycle. Results Probl Cell Differ. 53: 31-73.

5. Dubash AD, Wennerberg K, García-Mata R, Menold MM, Arthur WT, Burridge K. (2007) A novel role for Lsc/p115 RhoGEF and LARG in regulating RhoA activity downstream of adhesion to fibronectin. J Cell Sci. 120: 3989-3998.

6. Egea G, Serra-Peinado C, Gavilan MP, Rios RM (2015) Cytoskeleton and Golgiapparatus interactions: a two-way road of function and structure. Cell Health and Cytoskeleton, 7: 37-54

7. Geissmann F, Manz MG, Jung S, Sieweke MH, Merad M, Ley K. (2010). Development of Monocytes, Macrophages, and Dendritic Cells. Science 327: 656-661 8. Gilman AG. (1987). G proteins: transducers of receptor-generated signals. Ann. Rev. Biochem. 56: 615-49.

9. Hettinger J, Richards DM, Hansson J, Barra MM, Joschko AC, Krijgsveld J, Feuerer M. (2013) Origin of monocytes and macrophages in a committee progenitor. Nat Immunol. 14(8): 821-30.

10. Hoff M (2014). A real stretch: mechanisms behind cell elongation. PLoS Biol. 12(2):e1001782. 
11. Kloc M, LI XC, Ghobrial RM. (2014). RhoA cytoskeletal pathway to transplantation. J Immunol Clin Res 2: 1012.

12. Lee $\mathrm{CH}$, Kim YJ, Jang JH, Park JW. (2016) Modulating macrophage polarization with divalent cations in nanostructured titanium implant surfaces. Nanotechnology. $27: 085101$.

13. Lee K, Kim EH, Oh N, Tuan NA, Bae NH, Lee SJ, Lee KG, Eom CY, Yim EK, Park S. (2016). Contribution of actin filaments and microtubules to cell elongation and alignment depends on the grating depth of microgratings. J Nanobiotechnology. 14: 3514. Lin BH, Tsai MH, Lii CK, Wang TS. (2016) IP3 and calcium signaling involved in the reorganization of the actin cytoskeleton and cell rounding induced by cigarette smoke extract in human endothelial cells. Environ Toxicol. 31:1293-1306.

15. Liu Y, Minze LJ, Mumma L, Li XC, Ghobrial RM, Kloc M. (2016a) Mouse macrophage polarity and ROCK1 activity depend on RhoA and non-apoptotic Caspase3. Exp Cell Res 341:225-236.

16. Liu Y, Tejpal N, You J, Li XC, Ghobrial RM, Kloc M (2016b). ROCK inhibition impedes macrophage polarity and functions. Cell Immunol 300:54-62.

17. Liu Y, Chen W, Minze LJ, Kubiak JZ, Li XC, Ghobrial RM, Kloc M. (2016c) Dissonant response of M0/M2 and M1 bone-marrow-derived macrophages to RhoA pathway interference. Cell Tissue Res. 366:707-720

18. Liu Y, Chen W, Wu C, Minze LJ, Kubiak JZ, Li XC, Kloc M, Ghobrial RM. (2017) Macrophage/monocyte-specific deletion of Ras homolog gene family member A (RhoA) downregulates fractalkine receptor and inhibits chronic rejection of mouse cardiac allografts. J Heart Lung Transplant: 36:340-354.

19. Marra P, Salvatore L, Mironov A Jr, Di Campli A, Di Tullio G, Trucco A, Beznoussenko G, Mironov A, De Matteis MA. (2007). The biogenesis of the Golgi 
ribbon: the roles of membrane input from the ER and of GM130. Mol Biol Cell 18:1595608

20. Maxfield FR and McGraw TE. (2004). Endocytic recycling. Nature Reviews Mol. Cell Biol. 5: 121-132

21. McWhorter FY, Wang T, Nguyen P, Chung T, Liu WF. (2013) Modulation of macrophage phenotype by cell shape. Proc Natl Acad Sci U S A. 110: 17253-8

22. Mori T, Takahashi K, Naito M, Kodama T, Hakamata H, Sakai M, Miyazaki A, Horiuchi S, Ando M. (1994). Endocytic pathway of scavenger receptors via trans-Golgi system in bovine alveolar macrophages. Lab Invest. 71: 409-416.

23. Murray PJ, Wynn TA. (2011) Protective and pathogenic functions of macrophage subsets. Nat Rev Immunol. 11: 723-37

24. Nakamura N. (2010). Emerging new roles of GM130, a cis-Golgi matrix protein, in higher order cell functions. J Pharmacol Sci 112: 255-264.

25. Prosser DC, Wendland B. (2012). Conserved roles for yeast Rho1 and mammalian RhoA GTPases in clathrin-independent endocytosis. Small GTPases. 3: 229-235.

26. Rossanese OW, Reinke CA, Bevis BJ, Hammond AT, Sears IB, O'Connor J, Glicka BS (2001) A Role for Actin, Cdc1p, and Myo2p in the Inheritance of Late Golgi Elements in Saccharomyces cerevisiae. J Cell Biol. 153: 47-62.

27. Shang X, Marchioni F, Evelyn CR, Sipes N, Zhou X, Seibel W, Wortman M, and Zhenga Y. (2013) Small-molecule inhibitors targeting G-protein-coupled Rho guanine nucleotide exchange factors Proc Natl Acad Sci U S A. 110: 3155-3160.

28. Shang X, Marchioni F, Sipes N, Evelyn CR, Jerabek-Willemsen M, Duhr S, Seibel W, Wortman M, Zheng Y (2012). Rational design of small molecule inhibitors targeting RhoA subfamily Rho GTPases. Chem Biol. 19: 699-710.] 
29. Wang T, Luu TU, Chen A, Khine M, Liu WF. (2016) Topographical modulation of macrophage phenotype by shrink-film multi-scale wrinkles. Biomater Sci. 4:948-52.

30. Wheeler AP, Ridley AJ. (2004) Why three Rho proteins? RhoA, RhoB, RhoC, and cell motility. Exp Cell Res 301:43-9.

31. Zilberman Y, Alieva NO, Miserey-Lenkei S, Lichtenstein A, Kam Z, Sabanay H, Bershadskya A. (2011). Involvement of the Rho-mDia1 pathway in the regulation of Golgi complex architecture and dynamics. Mol Biol Cell. 22: 2900-2911.

32. SeemannJ, Jokitalo E, Pypaert M, Warren G. (2000). Matrix proteins can generate the higher order architecture of the Golgi apparatus. Nature, 407:1022-1026.

33. Kloc M, Kubiak JZ, Li XC, Ghobrial RM. (2014). The newly found functions of MTOC in immunological response. J Leukoc Biol. 95:417-30. 


\section{Figure Legends}

Figure 1. Rho-GEFs inhibition effect on wild type MO, M1 and M2 macrophage shape. Macrophages were stained with rhodamine-phalloidin to visualize actin (red) and DAPI to visualize nuclei (blue). (A, A1, A2) GEFs inhibition elongates MO macrophages. (B, B1, B2) GEFs inhibition does not influence M1 macrophage phenotype. (C, C1a, C1b, C2a, C2B) GEFs inhibition elongates majority of M2 macrophages and some macrophages remain slightly elongated or round. All are merged images of actin staining and DAPI. Bar in A, B, B1, B2 is equal to $50 \mu \mathrm{m}$ and $\mathrm{A} 1-\mathrm{A} 2$, and $\mathrm{C}-\mathrm{C} 2 \mathrm{~b}$ to $100 \mu \mathrm{m}$.

Figure 2. Quantification of the effect of Rho-GEFs inhibition on macrophage shape

A) Graph of quantitative distribution of particular macrophage shapes in control and treated MO macrophages; inhibitor treatment causes elongation and multipolarity of MO macrophages/ B) Graph of quantitative distribution of particular macrophage shapes in control and treated M1 macrophages; inhibitor treatment does not affect shape of M1 macrophages. C) Graph of quantitative distribution of particular macrophage shapes in control and treated M2 macrophages; inhibitor treatment causes extreme elongation and multipolarity of M2 macrophages. Graphs represent data from 3 independent experiments. The numbers of cells represent the combined numbers of cells with particular phenotype from three separate slides from three independent experiments within five different $0.05 \mathrm{~mm}^{2}$ area on each slide.

Figure 3. Quantification of the effect of Rho-GEFs inhibition on the frequency of different macrophage phenotype 
A) Graph of frequency of particular macrophage phenotype in control and treated MO macrophages B) Graph of frequency of particular macrophage phenotype in control and treated M1 macrophages. C) Graph of frequency of particular macrophage phenotype in control and treated M2 macrophages. Graphs represent data from 3 independent experiments. The frequencies represent the combined numbers of cells with particular phenotype from three separate slides from three independent experiments within five different $0.05 \mathrm{~mm}^{2}$ area on each slide. D) The comparison of the number of $\mathrm{M} 2$ macrophages with extremely elongated phenotype from three independent rhosin treatment experiments. The graph shows that there is no statistically significant difference between 3 different experiments. E) The comparison of number of M2 macrophages with multipolar phenotype from three independent rhosin treatment experiments. The graph shows that there is no statistically significant difference between 3 different experiments. The number of cells with given phenotype was calculated under $20 \mathrm{X}$ objective in two different $15 \mathrm{~mm}^{2}$ areas of the slide in each experiment.

Figure 4. Summary of the effects of Rho-GEFs inhibition on peritoneal MO, M1 and M2 macrophages

\section{Figure 5. Expression of M1 and M2 specific markers}

A) Western blot analysis of expression of M1 specific marker iNos in untreated and GEFs inhibitors treated MO, M1 and M2 macrophages. B) Graph representation of results from 3 independent Western blots showing lack of statistically significant difference in the expression of iNos between control and $Y 1$ or Rhosin treated macrophages. C) Western blot analysis of expression of M2 specific marker Arg-1 in untreated and GEFs inhibitors treated MO, M1 and M2 macrophages. B) Graph 
representation of results from 3 independent Western blots showing lack of statistically significant difference in the expression of Arg-1 between control and $\mathrm{Y} 1$ or Rhosin treated macrophages. GAPDH was used as a loading control.

\section{Figure 6. Effect of Rho-GEFs inhibition on macrophage Golgi complex}

Wild type macrophages immunostained with Golgi marker anti-GM130 antibody and FITC conjugated secondary antibody (green), and counterstained with rhodaminephalloidin for actin (red) and DAPI for nuclei (blue). A-A2) M0, B-B2) M1 and C-C3) M2 macrophages show diminution of the compact Golgi and slight dispersion of GM130 staining after GEFs inhibition. In addition M2 macrophages show translocation of GM130 protein toward macrophage tail after GEFs inhibition. All images showing Golgi and nucleus are merged images of GM130 staining and DAPI. Bar is equal to $62 \mu \mathrm{m}$.

\section{Figure 7. Effect of RhoA deletion on macrophage Golgi complex}

RhoA-deleled macrophages immunostained with Golgi marker anti-GM130 antibody and FITC conjugated secondary antibody (green), and counterstained with DAPI for

nuclei (blue). A) M0 KO macrophages and B) M1 KO macrophages show diminution of the compact Golgi. (C, D) The Rho KO M2 macrophages show diminution of Golgi and redistribution of GM130 protein toward macrophage front, tail and filopodia. All images showing Golgi and nucleus are merged images of GM130 staining and DAPI. Bar is equal to $100 \mu \mathrm{m}$

Figure 8. Quantification of the effect of Rho-GEFs inhibition on Golgi complex A) The effect of GEFs inhibition on Golgi compactness as assessed by the size (in $\mu \mathrm{m}$ ) of GM130 positive area in MO and M2 wild type macrophages. B) Comparison of the 
size (in $\mu \mathrm{m}$ ) of the compact Golgi in the vicinity of nucleus in control and RhoA-deleted (RhoA KO) MO and M2 macrophages. The measurements represent the combined numbers from three separate slides from three independent experiments.

C) The effect of RhoA deletion on the distance (measured in $\mathrm{nm}$ ) between the nucleus and the Golgi. In the wild type MO and M2 macrophages Golgi was situated at $309.18 \pm$ $184.2 \mathrm{~nm}$ distance from the nucleus. In RhoA deleted macrophages this distance was over 4 times larger i.e. $1305.95 \pm 558.6 \mathrm{~nm}$. This difference was statistically significant with $p<0.0001$. For electron microscopy analysis each grid from each experiment contained 100 cells. The cells were examined at electron microscopy level and 2-3 representative cells out of 100 from each grid were used for the measurements. The distance between nuclear membrane and first recognizable Golgi cisternae was measured on electron microscopy images.

Figure 9. The Golgi complex ultrastructure. Transmission electron microscopy of Golgi complex in wild type, RhoA-deleted and GEFs inhibited macrophages. All images are the examples of M2 macrophages. A) In wild type macrophages Golgi complex form multiple stacks of flat membranous cisternae (arrows) in the vicinity of the nucleus $(\mathrm{N})$. B) In RhoA-deleted macrophages only few Golgi cisternae (arrows) remain. C, D) GEFs inhibition results in disappearance of the majority of Golgi cisternae. The remnants of the still recognizable Golgi cisternae are indicated by arrows. Bar is equal to $500 \mathrm{~nm}$.

\section{Figure 10. Electron microscopy of centriole in wild type and RhoA deleted} macrophages

A) Low magnification of wild type MO macrophage showing the position of centriole (arrow). B) High magnification of the centriole region of macrophage shown in panel A. Centriole is surrounded by Golgi cisternae. C) Low magnification of M2 RhoA deleted 
macrophage showing the position of centriole (arrow). D) High magnification of the centriole region of macrophage shown in panel C. Centriole is surrounded by Golgi free cytoplasm and remnants of Golgi. Mito-mitochondria. Bar in panel A is equal to $2 \mu \mathrm{m}$, in panel $B$ to $500 \mathrm{~nm}$, in panel $\mathrm{C}$ to $10 \mu \mathrm{m}$ and in panel $\mathrm{D}$ to $500 \mathrm{~nm}$.

Figure 11. Summary of the effect of Rho-GEFs inhibition on M0, M1 and M2 macrophages

GEFs bind to RhoA and facilitate exchange of GDP to GTP and RhoA activation. Rhosin blocks GEFs binding site in RhoA, and $\mathrm{Y} 16$ blocks RhoA binding site in several GEFs including LARG, p115, and PDZ.

Figure 12. Effect of Rho-GEFs inhibition, and RhoA deletion on Golgi complex and Golgi-dependent functions.

Proper organization of the Golgi complex depends on actin cytoskeleton, which is regulated by RhoA pathway. Golgi complex buds off membranous vesicles: Iysosomes, endosomes, and transport/secretory vesicles that participate in receptor recycling and phagocytosis. RhoA pathway interference (RhoA deletion, RhoA/ROCK inhibition or GEFs inhibition) disrupts RhoA function. This in turn disrupts actin cytoskeleton, Golgi structure and function of Golgi-derived vesicles and functions. 


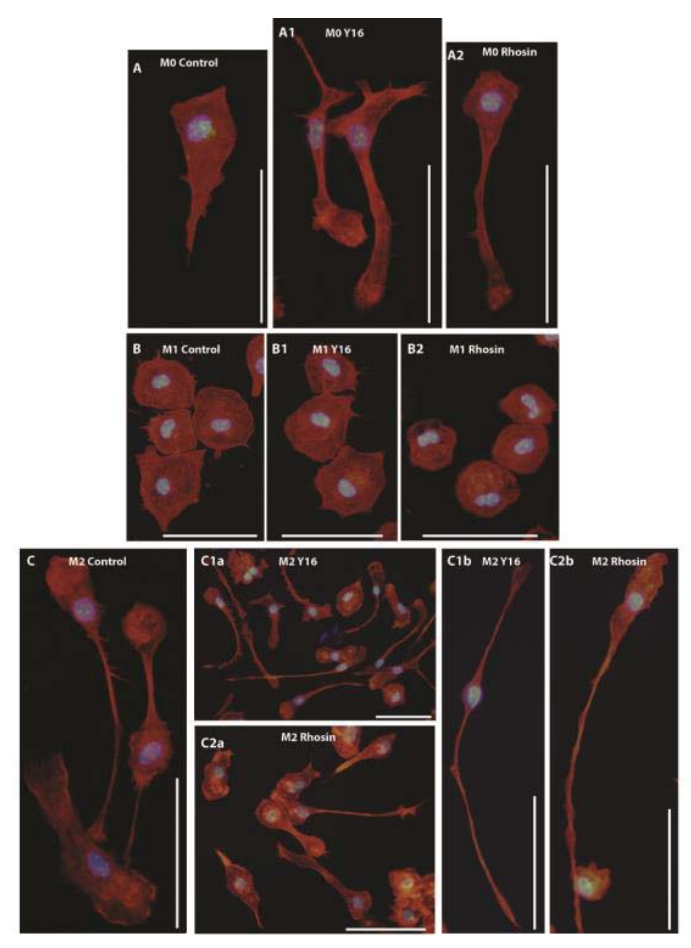


A

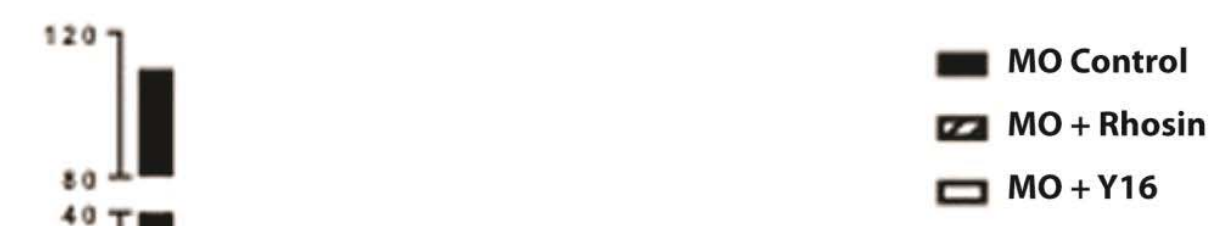

B
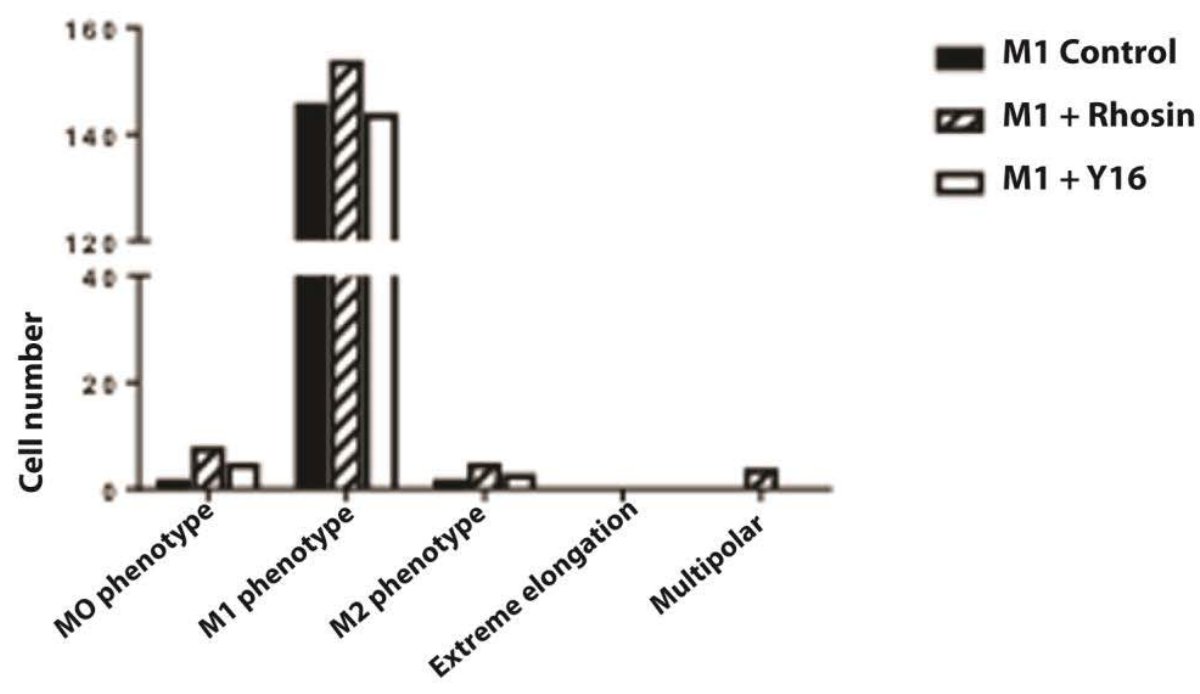

C

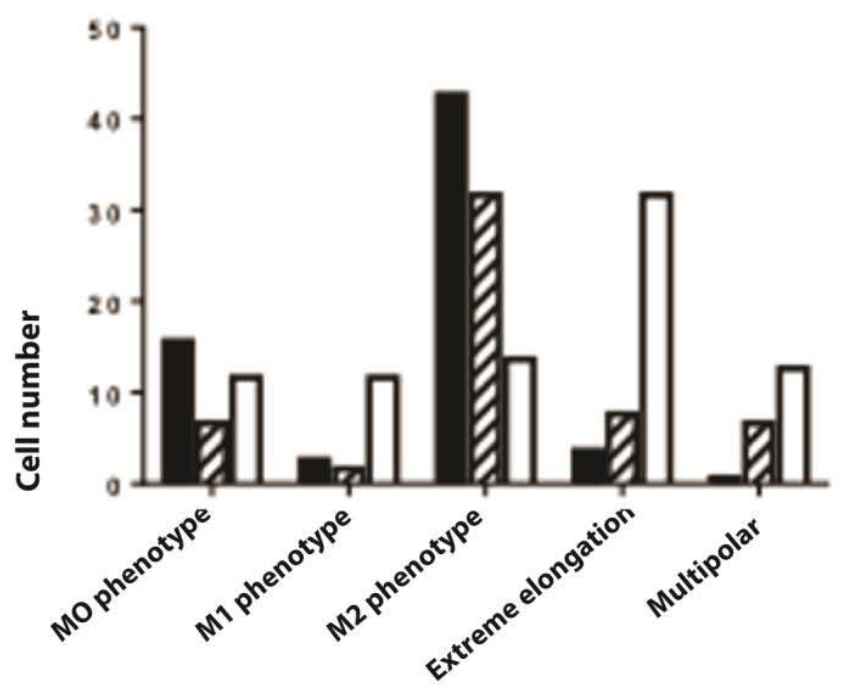


A

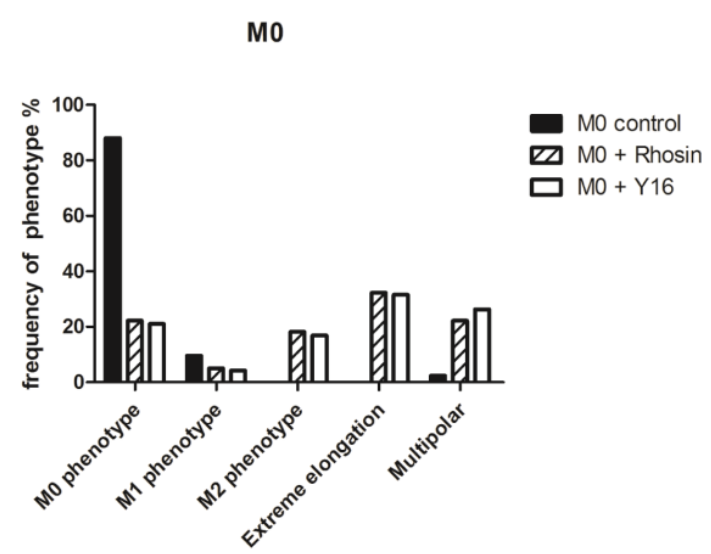

C

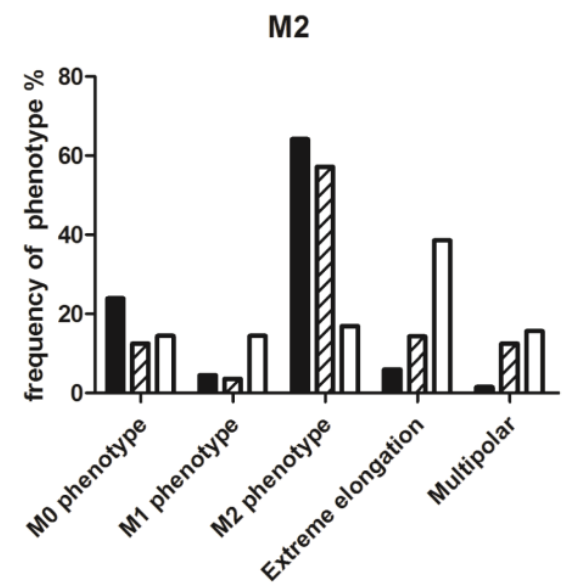

B

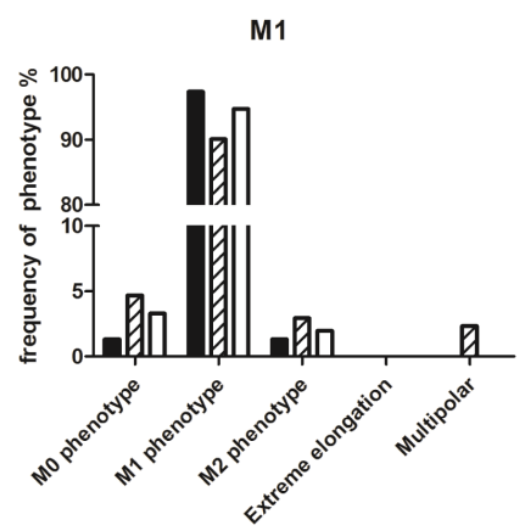

M1 control

ש7 $\mathrm{M} 1+$ Rhosin

$\square \mathrm{M} 1+\mathrm{Y} 16$
D

\section{Extremely elongated M2}

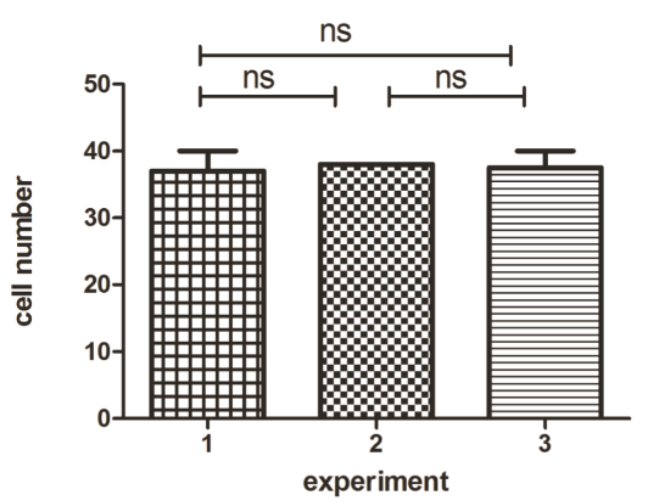

E

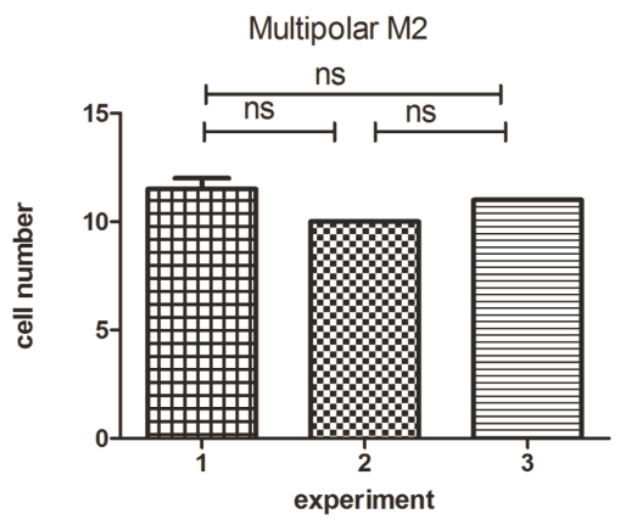




\section{Peritoneal Macrophages}

\begin{tabular}{|c|c|c|}
\hline Initial phenotype & Treatment & Resulting phenotype \\
\hline \multirow{5}{*}{ Mo } & LPS and IFN-y & M1 \\
\hline & $\begin{array}{l}\text { LPS and IFN-Y } \\
+ \text { Y16 } \\
\text { LPS and IFN-y } \\
\text { + Rhosin }\end{array}$ & \\
\hline & IL-4 and IL-13 & \\
\hline & $\begin{array}{l}\text { Y16 alone } \\
\text { Rhosin alone } \\
\text { RhoA deletion } \\
\text { ROCK inhibition }\end{array}$ & \\
\hline & $\begin{array}{l}\text { IL-4 and IL-13 } \\
+ \text { Y16 } \\
\text { IL-4 and IL-13 } \\
\text { + Rhosin }\end{array}$ & \\
\hline
\end{tabular}

A

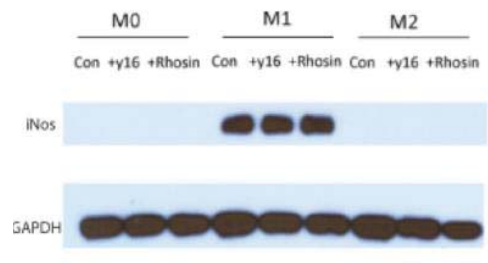

C

$\frac{M 0}{\text { Con }+ \text { y16 +Rhosin }} \frac{\text { M1 }}{\text { Con ty16 +Rhosin }} \frac{\text { M2 }}{\text { con ty16 +Rhosii }}$

Arg1

GAPOH
B
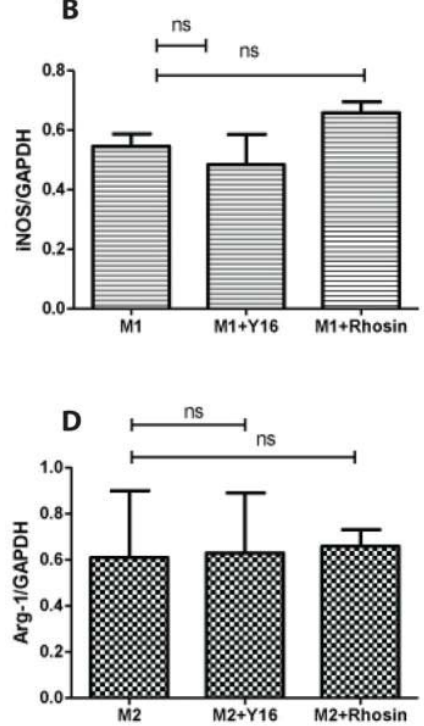

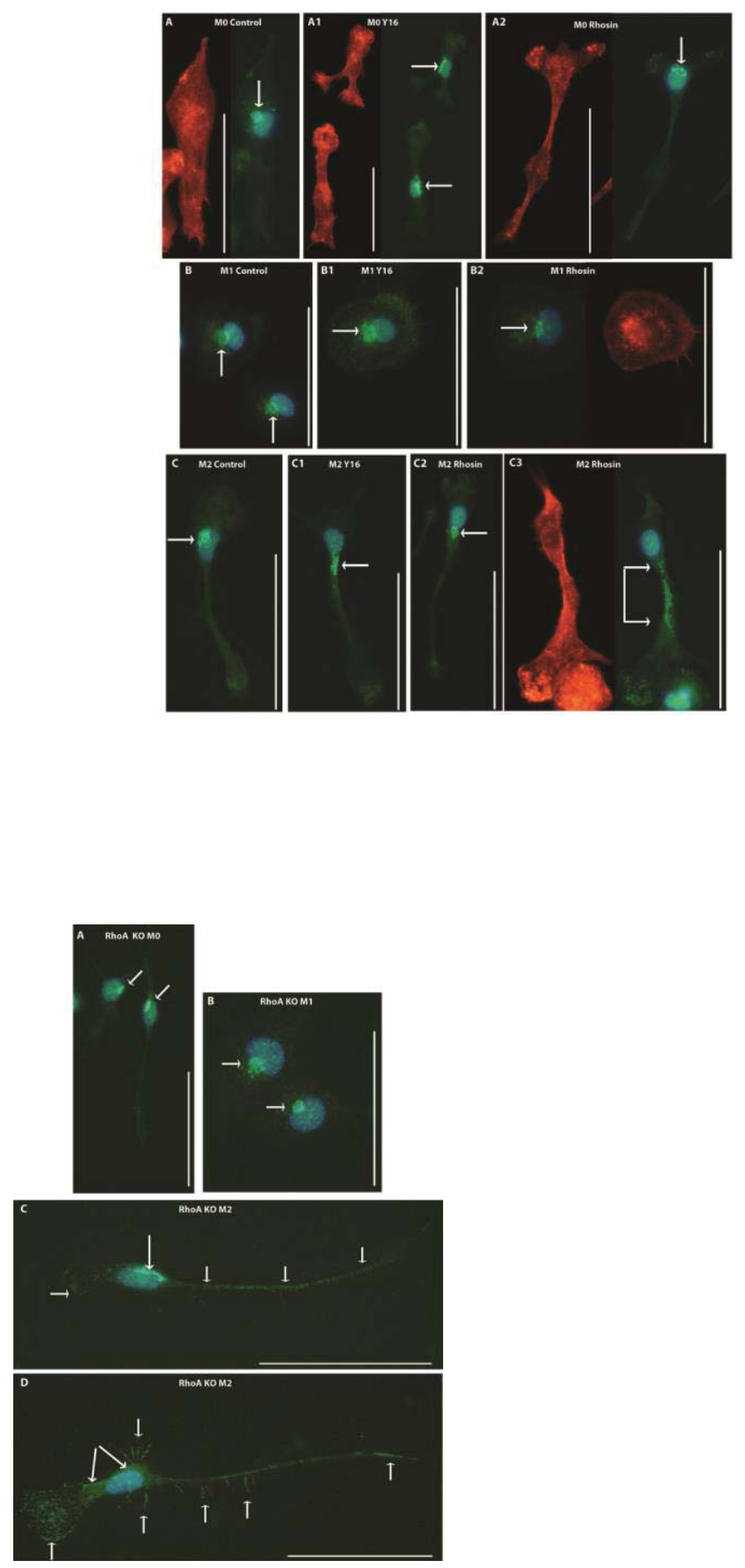
A

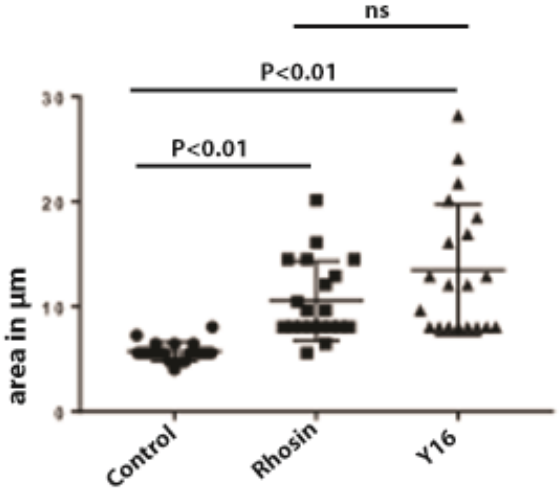

B

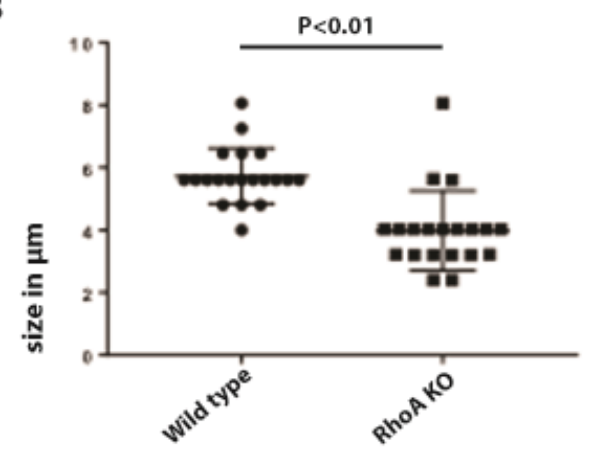

C

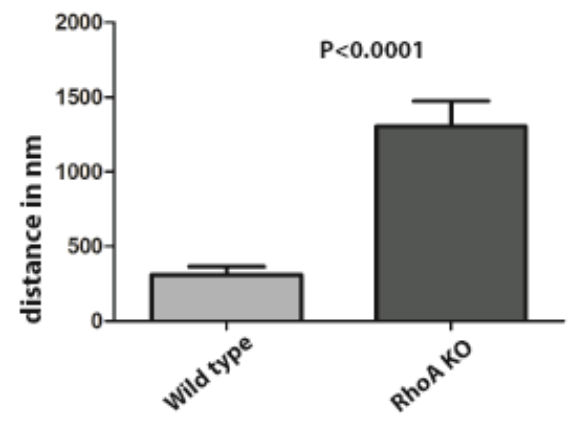




\section{ACCEPTED MANUSCRIPT}
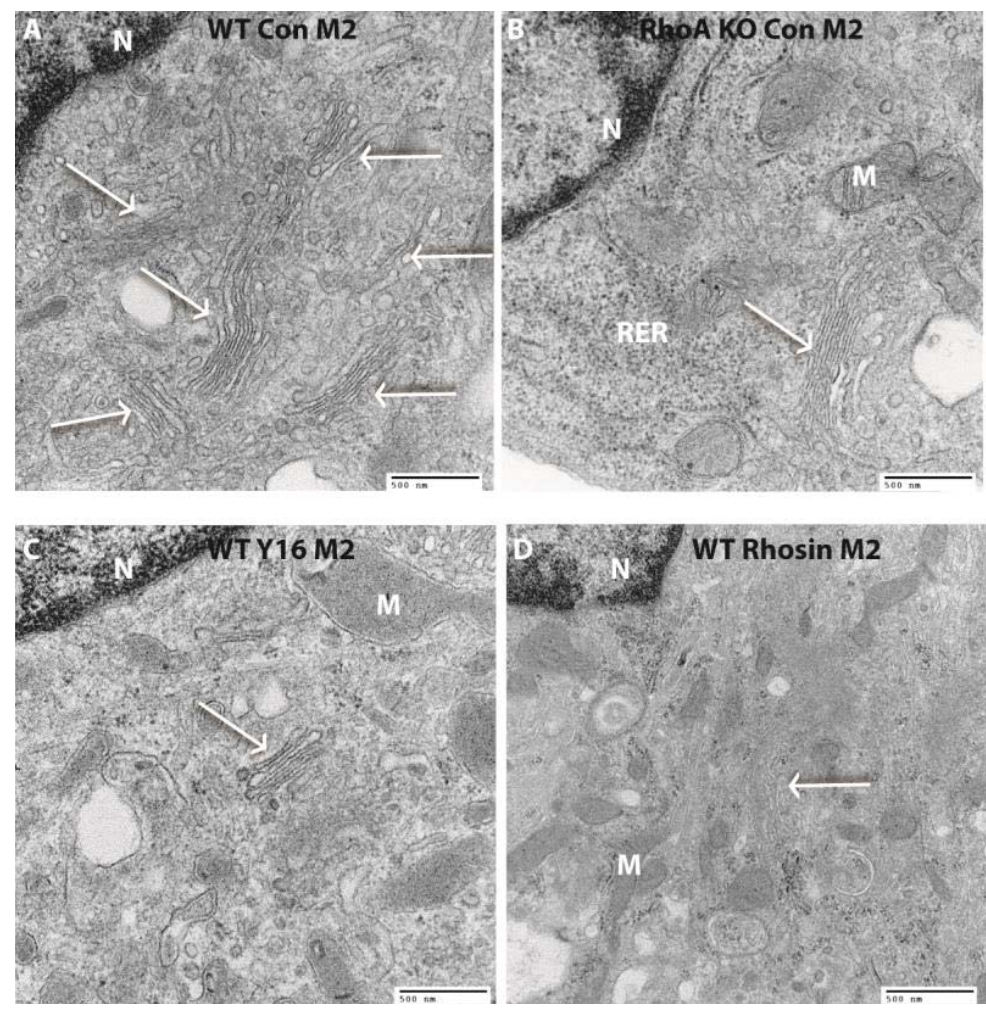


\section{ACCEPTED MANUSCRIPT}
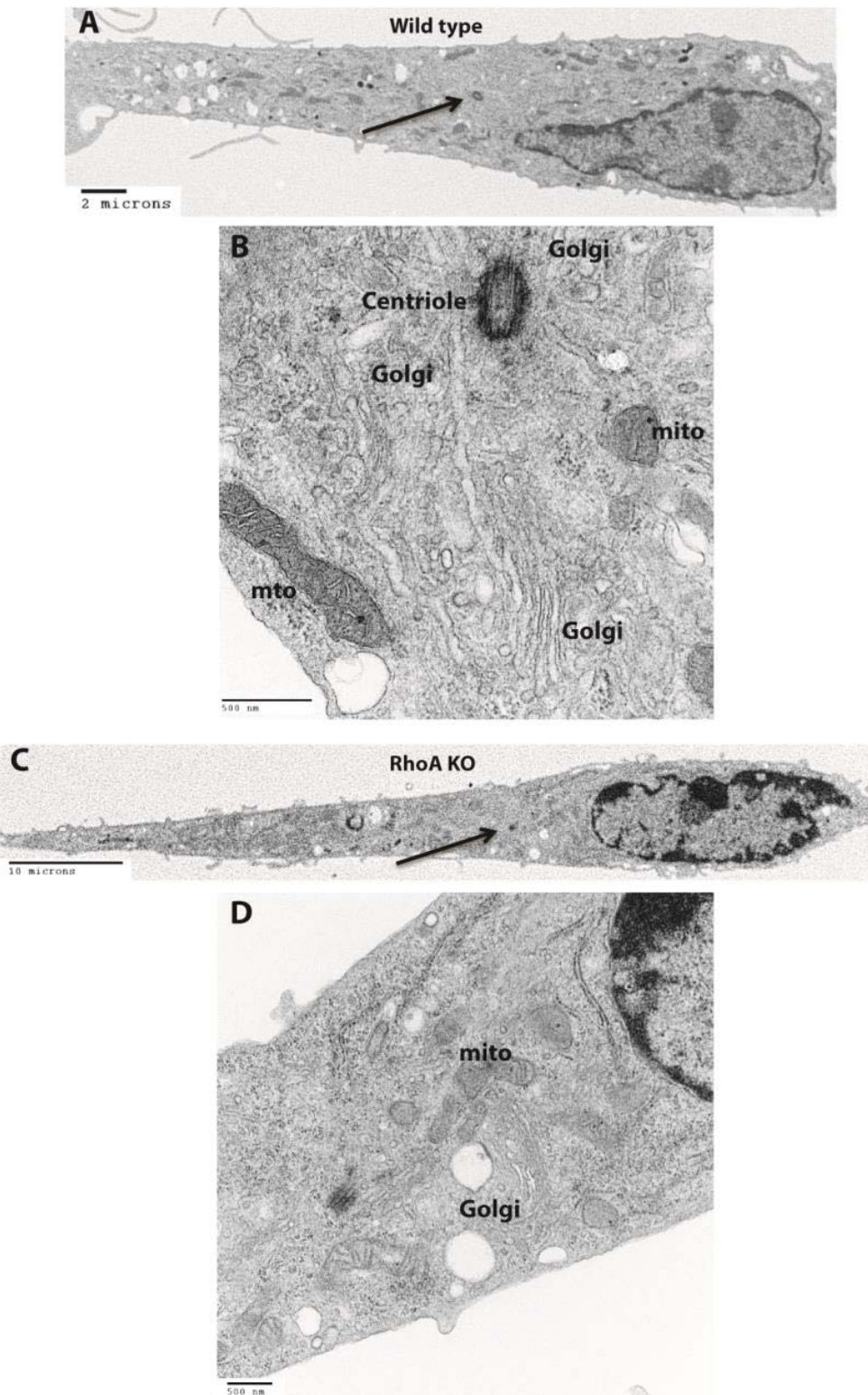

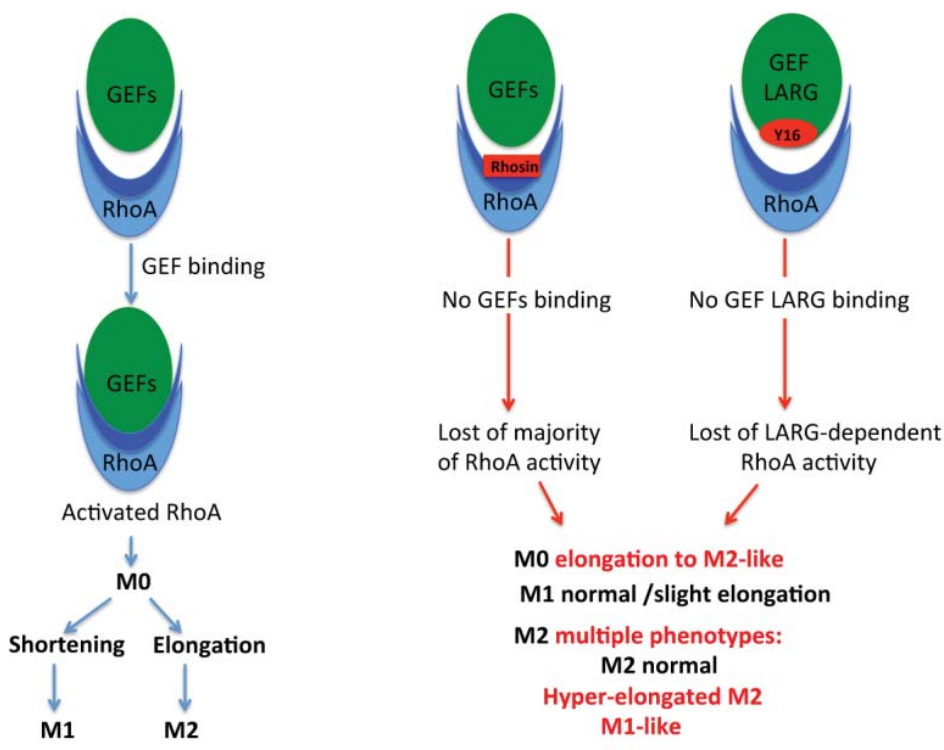

M0 elongation to M2-like

M1 normal /slight elongation

M2 multiple phenotypes: M2 normal

Hyper-elongated M2 M1-like

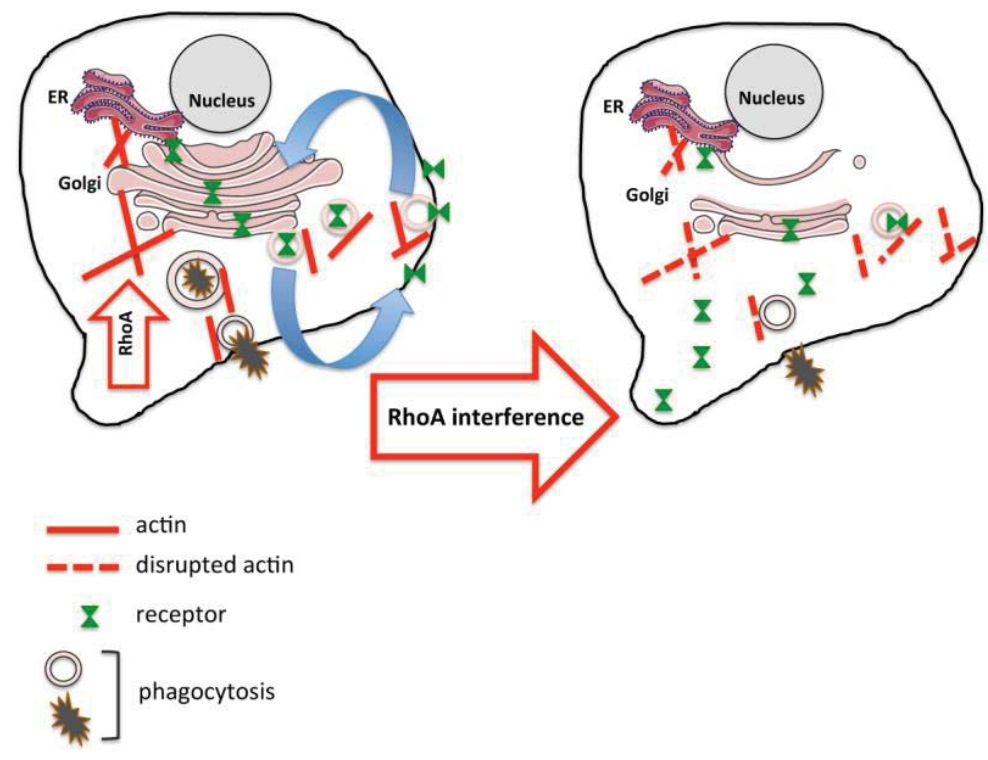

